\title{
Axon radius measurements in vivo from diffusion MRI: a feasibility study
}

\author{
Daniel C. Alexander \\ Department of Computer Science and Centre for Medical Image Computing \\ UCL (University College London) \\ Gower Street, London, WC1E 6BT, UK \\ D.Alexander@cs.ucl.ac.uk
}

\begin{abstract}
This paper investigates the feasibility of using diffusion MRI to measure axon-cell dimensions in the white matter of live subjects. A simple geometric model of white-matter tissue provides an expression that relates the axon radius to the diffusion MRI signal. The aim is to determine the accuracy and precision with which we can estimate this potentially important new biomarker. Precision and accuracy depend critically on the acquisition protocol. The paper proposes a general strategy to optimize the experiment design of in-vivo diffusion MRI experiments. The applicability of the design optimization extends well beyond the current work to optimizing the acquisition for any model of the diffusion process. Simulation experiments and results suggest feasibility of measuring larger axon radii in vivo on modern MRI scanners using the optimized acquisition schemes, but that higher gradient strengths are required to measure smaller axons.
\end{abstract}

\section{Introduction}

Diffusion MRI measures the displacement of particles, usually water molecules, within a material over a fixed time interval. The material microstructure determines the scatter pattern of particles within. Hence, measurements of the particle displacement reveal information about the microstructure. The standard diffusion MRI technique is Diffusion Tensor (DT) MRI [7], which assumes a Gaussian model of the particle displacements. DT-MRI provides two unique insights into material microstructure. First, it provides quantitative measurements of the anisotropy of particle displacements and, second, it provides an estimate of their dominant orientation. In fibrous material, such as white matter in the brain, the dominant orientations of particle displacements reflect the dominant fibre directions. Tractography algorithms follow these fibre-orientation estimates from point to point to determine the connectivity of the whole brain. Connectivity mapping through tractography provides fundamental insights in basic neuroscience [8, 25], reveals differences in disease [15] and assists prognoses after brain injury, stroke [23] or surgery [27].

The diffusion anisotropy depends on the density and orientational coherence of axon fibers and is often used as an indicator of white-matter integrity. Simple biomarkers derived from the diffusion tensor, such as mean diffusivity (MD) and fractional anisotropy (FA), are useful as indicators of major microstructural changes, such as brain damage through stroke [30] and also correlate with cognitive ability $[9,34]$. These biomarkers also highlight diseased tissue (eg $[33,36])$ and allow monitoring of development [19] and aging [26].

A limitation of biomarkers derived from the diffusion tensor is that they are non-specific and do not capture more subtle effects that might be earlier indicators of disease. Changes in FA accompany changes in axon density, radius distribution, orientation distribution or permeability and cannot distinguish between them. Moreover, combinations of changes in these features may result in no FA change at all. Direct measurements of microstructural features would provide much greater insight into disease mechanisms, recovery and development. Such measurements would also provide basic neuroscientific insight and may predict cognitive performance better than non-specific markers like FA.

Previous work $[4,12,31,37]$ shows that diffusion MRI can provide estimates of features such as pore sizes in minerals and axon sizes, density and permeability in excised biological tissue. However, all previous techniques rely on both prior knowledge of the pore orientation and lengthy data acquisitions (of order days) with high magnetic-field gradient strengths. The techniques are therefore practical only on non-live samples. Measuring these features in vivo is broadly considered impossible. This work initiates a challenge to that belief. The work is a first step towards a longterm goal of constructing a diffusion MRI technique that can measure axon radii, and other direct microstructural features, in live brain tissue. For in-vivo imaging, we are 
limited to moderate gradient strengths, the axon orientation is unknown and the tolerable acquisition time is an order of magnitude lower (around half an hour). This paper is a feasibility study into measuring one particular feature, the axon radius. Simulation results show that, for a simple and idealized model, we can tune a 30-minute acquisition to enable recovery of the axon radii in the range found in the human brain $(0.25-10 \mu \mathrm{m})$ with unknown axon orientation. The result, and the methods developed to obtain it, opens doors to a new class of in-vivo diffusion MRI technique with a wide range of new applications.

The next section gives some background on diffusion MRI and how we use it to infer tissue microstructure. Section 3 introduces the simple geometric model of white matter tissue and derives the corresponding model for the diffusion MRI signal. It then introduces a general method for experiment design in diffusion MRI, which we can use to optimize the acquisition protocol for fitting the simple model. Section 4 evaluates the precision and accuracy with which we can estimate the axon radius using the optimized acquisition schemes. Section 5 concludes.

\section{Background}

This section covers essential background on diffusion MRI. In particular, the following subsections outline the basic pulse sequence for acquiring diffusion MRI measurements. We then discuss the nature of particle scattering in white matter and review modelling techniques in the literature that relate the diffusion MRI signal to features of material microstructure. The review leads to the development of a simple model for testing in section 3. Finally, we review previous methods in the literature that specifically measure axon radii using diffusion MRI for comparison with the new approach developed here.

\subsection{The measurement}

The standard pulse sequence for diffusion MRI is a pulsed-gradient spin-echo (PGSE) sequence, which places equal diffusion gradient pulses either side of a 180-degree radio-frequency pulse at the centre of the sequence. Figure 1 shows a diagram of the pulse sequence. The length $\delta$, strength and direction $\mathbf{G}$ and separation $\Delta$ of the gradient pulses all control the sensitivity of the signal to particle displacements. These are the three main tunable parameters of the sequence.

If $\delta \ll \Delta$, the normalized signal $A(\mathbf{q})$ approximates the Fourier transform of the distribution of particle displacements (ie the scatter pattern) $p$ at wavenumber $\mathbf{q}=\gamma \delta \mathbf{G}$, where $\gamma$ is the gyromagnetic ratio; see $[1,10]$. This Fourier relationship is the basis of most diffusion MRI methods. For example, in DT-MRI, $p$ is a zero-mean Gaussian with covariance $2 \mathrm{D} t$, where $\mathrm{D}$ is the $\mathrm{DT}$ and $t=\Delta$ is the diffusion

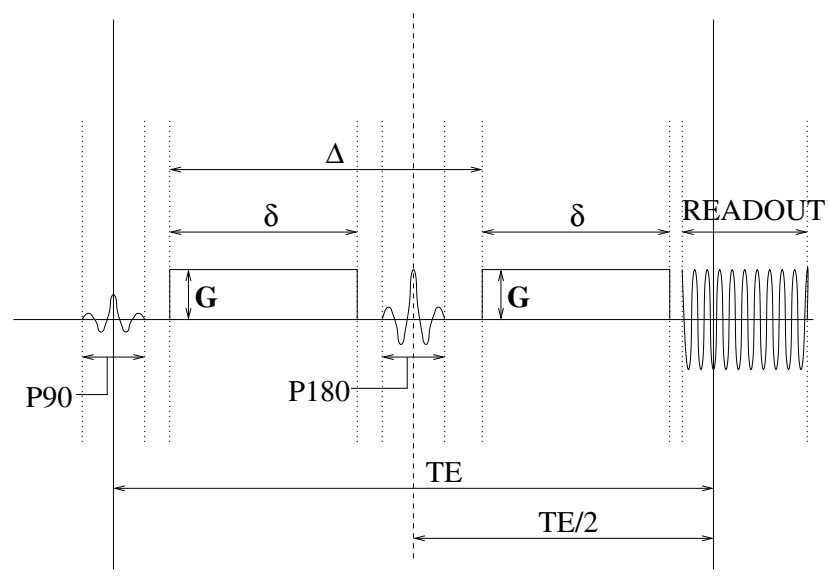

Figure 1. The pulsed-gradient spin-echo sequence.

time. From the Fourier transform, $A(\mathbf{q})=\exp \left(-t \mathbf{q}^{T} \mathbf{D q}\right)$, which is the standard equation for fitting the DT to measurements [7]. In practice, $\delta$ is rarely negligible. For simple Gaussian displacements, we can correct for finite $\delta$ by setting $t=\Delta-\delta / 3$ [10]. However, $p$ in tissue is not Gaussian and finite- $\delta$ effects are difficult to model [20].

\subsection{Diffusion in tissue}

Departures of $p$ from the Gaussian model in brain tissue are well documented in the literature, eg [31]. Specifically, the diffusion MRI signal does not decay "monoexponentially" as $|\mathbf{q}|$ and/or $t$ increase, as the Gaussian model predicts. The departure is no surprise. In white matter, for example, water molecules within axon cells exhibit restricted diffusion, since the axon's myelin sheath is largely impermeable to water over typical diffusion times. Other water molecules (around 20\%) are in the extracellular space and exhibit hindered diffusion. The extracellular space is normally mostly connected so water percolates and $p$ is approximately Gaussian [24]. Restricted intra-cellular diffusion, however, can produce highly non-Gaussian $p$, since cell dimensions limit the maximum possible displacement. The literature contains analytic models for $p$ within simple restricting geometries, such as spheres, cylinders and parallel planes $[11,22,29]$. These models relate simply to the diffusion MRI signal via the standard Fourier transform, but, as noted above, this fails to account for finite $\delta$. Murday and Cotts [21] derive a model for the diffusion MRI signal for particles diffusing within spheres that accounts for finite pulse widths by approximating the distribution of spin phases with a Gaussian. Their approach adapts easily to other simple geometries such as cylinders [35]. Synthetic measurements from molecular simulations agree closely with this approximation [6].

Various researchers construct geometric models from simple primitives, so that $p$ has analytic form, to try to 
match diffusion MRI signals more closely than the Gaussian model. For example, Szafer et al [32] construct a braintissue model consisting of non-abutting semi-permeable square cylinders. Stanisz et al's model [31] contains nonabutting spherical glial cells and ellipsoidal axons with semi-permeable walls embedded in a homogeneous substrate. Assaf and Basser's model [3] contains cylindrical non-abutting axons with a gamma distribution of radii. Sen and Basser [28] add thickness to cylinder walls (like myelin sheaths) and show that different parameter settings reflect the observations from brain tissue with various pathological conditions. Other models, eg [17], use similar primitives to construct models for $p$ in red blood cells.

\subsection{Axon Measurements}

A few researchers have used the modelling techniques from the previous section to measure axon radii. Assaf and Basser [4] acquire 1024 measurements: 8 repeats of each combination of $\Delta$ at 8 settings in the range $[0.02,0.15] \mathrm{s}$ and $|\mathbf{G}|$ at 16 settings in the range $[0,1.2] \mathrm{Tm}^{-1} ; \delta=0.0025 \mathrm{~s}$ throughout. The acquisition takes about 12 hours. They use ex-vivo nerve-tissue samples with known fibre orientation and set $\mathbf{G}$ perpendicular to the fibre direction for all the measurements. They model the distribution of axon radii with a Gamma distribution and show that the maximum likelihood distribution agrees closely with a histogram of radii measured by hand on a histological image of the same sample. Stanisz et al [31] use a similar acquisition and estimate axon density, radius and permeability in an excised bovine optic nerve.

Various non-model-based techniques aim to measure axon radii using the diffraction pattern of the diffusion MRI signal from restricted water molecules in the long-diffusiontime limit ( $\Delta \gg R^{2} / 2 d$, where $R$ is the pore radius and $d$ is the diffusivity) and assuming short gradient pulses $(\Delta \gg \delta)$. Callaghan et al [12] first noticed the diffraction pattern and related its frequency to the sample pore size. For example, Avram et al [5] recover the radii of cylindrical polymer tubes with radii $20 \mu \mathrm{m}$ and $9 \mu \mathrm{m}$ from the diffraction pattern. Weng et al [37] show that the diffraction pattern successfully determines the spacing between layers in a phantom constructed from piled acetates immersed in water. However, measurements from an excised rat brain fail to identify the axon radius measured by microscope in [37].

\section{Methods}

This section first develops a simple model for diffusion in white matter, then develops a general method for optimizing the experimental design of diffusion MRI acquisitions for estimating model parameters.

\subsection{Simple Model}

We use a model for white matter with two compartments between which there is no exchange of water molecules. The model assumes parallel non-abutting cylindrical axon cells with equal radii and impermeable walls embedded in a homogeneous extra-cellular medium. Water in the intracellular compartment exhibits restricted diffusion, but the intracellular medium is also homogeneous with the same diffusivity as the extracellular medium. Water in the extracellular compartment exhibits hindered diffusion. The normalized MRI signal is then

$$
A(\mathbf{G}, \Delta, \delta)=f A_{r}(\mathbf{G}, \Delta, \delta)+(1-f) A_{h}(\mathbf{G}, \Delta, \delta),
$$

where $f \in[0,1]$ is the fraction of water molecules in the intra-cellular compartment, $A_{r}$ and $A_{h}$ are the appropriately normalized signals from the intra and extra-cellular compartments, respectively.

We model the hindered extra-cellular diffusion as simple (Gaussian) anisotropic diffusion so that

$$
A_{h}(\mathbf{G}, \Delta, \delta)=\exp \left(-t \mathbf{q}^{T} \mathbf{D}_{h} \mathbf{q}\right),
$$

where we take the diffusion time $t=\Delta-\delta / 3, \mathrm{D}_{h}=\left(d_{\|}-\right.$ $\left.d_{\perp}\right) \mathbf{n} \mathbf{n}^{T}+d_{\perp} I$ is the diffusion tensor, which is cylindrically symmetric with major eigenvector $\mathbf{n}$ in the fibre direction with corresponding eigenvalue $d_{\|}$(the diffusivity parallel to $\mathbf{n}$ ) and minor eigenvalues $d_{\perp}$ (the apparent diffusivity perpendicular to $\mathbf{n}$ ), and $I$ is the identity tensor.

For the restricted component, we use Van Gelderen's extension [35] of Murday's model [21] for the MR signal from water diffusing in cylinders. We omit the lengthy expression here, but it comes directly from [35]. The model provides the MR signal $A_{r \perp}\left(G_{\perp}, \Delta, \delta\right)$ from water molecules inside a cylinder of radius $R$ for a gradient perpendicular to the fibre with strength $G_{\perp}$. In general, $\mathbf{G}$ is not perpendicular to $\mathbf{n}$, but we can decompose $\mathbf{G}$ into parallel $G_{\|}=|\mathbf{G}| \cos \theta$, where $\theta$ is the angle between $\mathbf{n}$ and $\mathbf{G}$, and perpendicular $G_{\perp}=|\mathbf{G}| \sin \theta$ components that attenuate the signal independently. We assume simple diffusion parallel to the fiber and that the intrinsic diffusion coefficient is $d_{\|}$, as in the extra-cellular compartment. Thus, the parallel component provides an additional attenuation

$$
A_{r \|}\left(G_{\|}, \Delta, \delta\right)=\exp \left(-t\left(\gamma \delta G_{\|}\right)^{2} d_{\|}\right) .
$$

The signal accounting for both components is then

$$
A_{r}(\mathbf{G}, \Delta, \delta)=A_{r \perp}\left(G_{\perp}, \Delta, \delta\right) A_{r \|}\left(G_{\|}, \Delta, \delta\right) .
$$

In summary, the parameters of the model are:

- The volume fraction of the intra-cellular compartment, $f$, which is equivalent to the axon density.

- The apparent diffusion coefficients, $d_{\|}$and $d_{\perp}$. 
- The fibre direction, $\mathbf{n}$.

- The axon radius, $R$.

This is an experimental model only and does not account for many of the microstructural variables in real brain tissue. Some other variables are straightforward to incorporate. We can include distributions of axon radii by integrating Eq. 4 over a prior on $R$, as in [4], which assumes a Gamma distribution of radii. Axon wall permeability allows exchange of particles between the two compartments, which we can model with a pair of coupled differential equations [31]. White matter also contains glial cells, which we might model as a third compartment with spherical restriction, as in [31]. We can allow distributions of fibre directions by integrating over a prior on $\mathbf{n}$. We may also be able to reduce the number of parameters in the model by one, by using models of tortuosity [24] to estimate $d_{\perp}$ from $d_{\|}, f$ and $R$. Other effects, such as abutting cells and loss of percolation of the extra cellular space are difficult to incorporate in analytic models.

\subsection{Sampling}

This section addresses experiment design, ie, the choice of measurements that give the most accurate and precise estimates of the fitted model parameters. The tunable settings of the PGSE pulse sequence are $\mathbf{G}, \Delta$ and $\delta$. Each measurement may use a different combination. We seek the set of combinations that give the best parameter estimates.

\subsubsection{In-vivo imaging}

In-vivo imaging places three key constraints on the acquisition sequence. First, we must limit the number of measurements to ensure the acquisition time is tolerable for live subjects. Here we use a limit of 120 measurements, which modern scanners can acquire in around 30 minutes. Second, both power and safety constraints limit the maximum gradient strength we can use to about $0.07 \mathrm{~T} \mathrm{~m}^{-1}$ for human subjects. Third, in general we have no prior knowledge about the orientation of axons, so we require an acquisition that allows estimation of the model parameters for arbitrary $\mathbf{n}$. In previous studies into measuring axon radii, $\mathbf{n}$ is assumed known since the orientation of excised tissue is controllable. Experiments use gradients oriented perpendicular to $\mathbf{n}$, which is assumed to give the most information about the intracellular compartment. To handle arbitrary fibre directions, we must acquire measurements with various gradient directions. In a similar way, DT-MRI commonly uses "high-angular resolution" acquisition schemes in which $|\mathbf{G}|, \Delta$ and $\delta$ are the same for each measurement, but each has a unique gradient direction with the whole set distributed evenly on a hemisphere [16].
The class of scheme we investigate here acquires the same number $M$ of measurements in each of $N$ gradient directions. The $M$ combinations of $|\mathbf{G}|, \delta$, and $\Delta$ are the same in each direction. We choose the $N$ directions by electrostatic minimization $[14,16]$, fix them, and optimize the $M$ combinations of $|\mathbf{G}|, \delta$, and $\Delta$.

\subsubsection{Objective Function}

The Fisher information matrix and the Cramer-Rao lower bound (CRLB) [18] are standard tools in experimental design. The CRLB provides a lower bound on the variance of a fitted model parameter that often correlates closely with the true variance.

To optimize the acquisition, we aim to minimize, with respect to the set of $|\mathbf{G}|, \delta$, and $\Delta$ combinations, the sum of the standard errors of each model parameter

$$
\tilde{F}=\sum_{i=1}^{K} \sigma_{i}^{2} / p_{i}^{2}
$$

where $p_{i}, i=1, \cdots, K$, are the model parameters and $\sigma_{i}$ is the standard deviation of $p_{i}$. We do not know the $\sigma_{i}^{2}$, so we use the CRLBs in their place. The general form of the Fisher information matrix $J$ has $(i, j)$-th element

$$
J_{i j}=E\left(\frac{\partial^{2} L}{\partial p_{i} \partial p_{j}}\right)
$$

where $L$ is the log likelihood given an appropriate noise model and $E$ denotes expectation given that noise model. For Gaussian noise,

$$
J_{i j}=\sigma^{-2} \sum_{k=1}^{N M}\left(\frac{\partial A}{\partial p_{i}} \frac{\partial A}{\partial p_{j}}\right)\left(\left(p_{n}\right)_{n=1}^{K} ; \mathbf{G}_{k}, \Delta_{k}, \delta_{k}\right),
$$

which is simple to derive from Eq. 6 [18]. The CRLB for $p_{i}$ is the $i$-th diagonal element of $J^{-1}$, so we replace $\tilde{F}$ by

$$
F=\sum_{i=1}^{K}\left(J^{-1}\right)_{i i} / p_{i}^{2}
$$

The function $F$ provides the basis of an objective function that we can minimize with respect to the $M$ combinations of $|\mathbf{G}|, \delta$, and $\Delta$. However, $F$ depends on specific choices for the parameter values $p_{i}$, which take a range of values. The full objective function therefore integrates $F$ over prior distributions on each $p_{i}$. Here, we assume $\delta$ function priors on the model parameters $f, d_{\|}, d_{\perp}$ and $R$. In particular, we set $f=0.7, d_{\|}=1.7 \times 10^{-9} \mathrm{~m}^{2} \mathrm{~s}^{-1}$, and $d_{\perp}=0.2 \times 10^{-9} \mathrm{~m}^{2} \mathrm{~s}^{-1}$ throughout. The next section uses a variety of settings for the axon radius $R$.

We cannot use a $\delta$-function prior for $\mathbf{n}$, however, as we require good parameter estimates for arbitrary fibre orientation. For orientation independence, we average $F$ over a 
set of directions. One approach is to average $F$ over a large number of evenly distributed sample directions. To reduce computation times, we instead construct a set of worst case n and average $F$ over only them. To construct the set, we choose the first element at random and minimize $F$ to obtain an initial optimized scheme. We find the $\mathbf{n}$, in a set $S_{500}$ of 500 sample directions evenly distributed over the sphere, that has the largest $F$ with the optimized scheme. We add that $\mathbf{n}$ to the set, repeat the optimization and iterate until the $\mathbf{n} \in S_{500}$ with the largest $F$ is already in the set. The process usually converges with the set containing only three or four elements.

\subsubsection{Rician CRLB}

The noise on MRI measurements in not Gaussian, but Rician [13], so that

$$
P(\tilde{A})=\frac{\tilde{A}}{\sigma^{2}} I_{0}\left(\frac{A \tilde{A}}{\sigma^{2}}\right) \exp \left(-\frac{A^{2}+\tilde{A}^{2}}{2 \sigma^{2}}\right),
$$

where $I_{i}, i=0,1,2, \cdots$, are the modified Bessel functions of the first kind; $\tilde{A}$ and $A$ are the measurement and signal (predicted by the model), respectively, and we drop the dependence on the pulse-sequence parameters from the notation. The Rician noise model assumes the signal is the modulus of a complex measurement with zero-mean Gaussian noise, with variance $\sigma^{2}$, on each component. The log likelihood of a set of measurements comes from taking logs of Eq. 9 and summing over all measurements to obtain, dropping constant terms,

$$
L_{R}=\sum_{k=1}^{N M} \log I_{0}\left(\frac{A_{k} \tilde{A}_{k}}{\sigma^{2}}\right)-2 \log \sigma-\frac{A_{k}^{2}+\tilde{A}_{k}^{2}}{2 \sigma^{2}} .
$$

We can derive an alternative CRLB from the general expression for the Fisher information matrix in Eq. 6 that uses a Rician noise model. We need to compute the expectations of the second derivatives of $L_{R}$ with respect to the $p_{i}$ and $\sigma$. For example,

$$
E\left(\frac{\partial^{2} L_{R}}{\partial p_{j} \partial p_{k}}\right)=\int_{0}^{\infty} \frac{\partial^{2} L_{R}}{\partial p_{j} \partial p_{k}} P(\tilde{A}) \mathrm{d} \tilde{A} .
$$

The following expectations are straightforward to compute, although the algebra is lengthy and we omit the details here:

$$
\begin{gathered}
E\left(\frac{\partial^{2} L_{R}}{\partial p_{i} \partial p_{j}}\right)=\sum_{k=1}^{N M} \frac{1}{\sigma^{4}} \frac{\partial A_{k}}{\partial p_{i}} \frac{\partial A_{k}}{\partial p_{j}}\left(Z_{k}-A_{k}^{2}\right) \\
E\left(\frac{\partial^{2} L_{R}}{\partial p_{i} \partial \sigma}\right)=\sum_{k=1}^{N M} \frac{2 A_{k}}{\sigma^{5}} \frac{\partial A_{k}}{\partial p_{i}}\left(Z_{k}-A_{k}^{2}-\sigma^{2}\right) \\
E\left(\frac{\partial^{2} L_{R}}{\partial \sigma^{2}}\right)=\sum_{k=1}^{N M} \frac{4 A_{k}^{2}}{\sigma^{6}}\left(\frac{\sigma^{4}}{A_{k}^{2}}-\sigma^{2}-A_{k}^{2}+Z_{k}\right),
\end{gathered}
$$

where

$$
Z_{k}=\int_{0}^{\infty} A_{k}^{2} I_{1}^{2}\left(\frac{A_{k} \tilde{A}}{\sigma^{2}}\right) I_{0}^{-2}\left(\frac{A_{k} \tilde{A}}{\sigma^{2}}\right) P(\tilde{A}) \mathrm{d} \tilde{A} .
$$

The function $Z_{k}$ does not have closed form and we have to compute it numerically. In practice, we compute a lookup table of sampled values and use linear interpolation to estimate it during optimization. Andersson [2] derives the Rician CRLB for the specific case of the diffusion tensor model. The new expressions above are for the general case.

\subsubsection{Optimization}

Optimizations of this type are complicated by many local minima in the objective function. We use a stochastic optimization algorithm, SOMA (Self-Organizing Migratory Algorithm) [38] with default settings, to perform the minimization of $F$ averaged over sample $\mathbf{n}$. In each experiment, we repeat the optimization five times and pick the result with the smallest final value of the objective function.

During minimization, we enforce several constraints. First, $\Delta_{k} \geq \delta_{k}+\mathrm{P} 180$, where $\mathrm{P} 180$ is the length of the 180-degree pulse, which we set to $0.005 \mathrm{~s}$. Second, $\delta_{k} \geq 0$. Third, $0 \leq\left|\mathbf{G}_{k}\right| \leq G_{\max }$, where $G_{\max }$ is the maximum available gradient strength. We also need to account for changes to the echo time, TE, required for the sequence, since $\mathrm{T}_{2}$ effects reduce the signal more for longer TE, which increases the significance of the noise. To account for the effects of varying TE, we set $\sigma=\sigma_{0} \exp \left(\max _{k}\left(\Delta_{k}+\right.\right.$ $\left.\left.\delta_{k}\right) / \mathrm{T}_{2}\right)$ in the Fisher information matrix in Eqs. 6 and 12, where $\sigma_{0}$ is the actual noise level. We set $\mathrm{T}_{2}=0.07 \mathrm{~s}$, which is typical for white matter, and assume a base setting of TE $=0.1 \mathrm{~s}$. Thus, if $\sigma_{0}=0.02$, the signal to noise ratio $(\mathrm{SNR})$ of the unweighted signal is 50 when $\mathrm{TE}=0.1 \mathrm{~s}$.

\section{Experiments and Results}

This section contains some experiments and results that test the feasibility of measuring $R$ using experimental design procedures from the previous section. All experiments use the simple model of section 3.1 and we use only synthetic data throughout. The experiments test two settings of $G_{\max }$. One setting is $G_{\max }=0.07 \mathrm{Tm}^{-1}$, which is at the limit of what modern human scanners can achieve on live subjects. The other is $G_{\max }=0.2 \mathrm{Tm}^{-1}$, which is easily achievable in small-bore animal scanners.

\subsection{Optimized acquisition schemes}

First, we compare qualitatively some acquisition schemes that the optimization procedure in section 3.2 produces. For illustration, we set $R=2 \mu \mathrm{m}, G_{\max }=$ $0.2 \mathrm{Tm}^{-1}, \sigma_{0}=0.02, N=30$ and $M=4$. Table 1 shows the optimized acquisition scheme for the objective 


\begin{tabular}{||l|l|l||l||}
\hline$|\mathbf{G}| / \mathrm{T} \mathrm{m}^{-1}$ & $\Delta / \mathrm{s}$ & $\delta / \mathrm{s}$ & $b / \mathrm{s} \mathrm{mm}^{-2}$ \\
\hline 0.200 & 0.025 & 0.020 & 20087 \\
0.200 & 0.026 & 0.018 & 18771 \\
0.121 & 0.029 & 0.016 & 6035 \\
0.200 & 0.013 & 0.008 & 1744 \\
\hline
\end{tabular}

Table 1. Optimized combinations of $|\mathbf{G}|, \delta$ and $\Delta$ from the Gaussian CRLB objective function. The table includes the more familiar quantity $b=t|\mathbf{q}|^{2}$, where $t=\Delta-\delta / 3$.

\begin{tabular}{||l|l|l||l||}
\hline$|\mathbf{G}| / \mathrm{T} \mathrm{m}^{-1}$ & $\Delta / \mathrm{s}$ & $\delta / \mathrm{s}$ & $b / \mathrm{s} \mathrm{mm}^{-2}$ \\
\hline 0.200 & 0.024 & 0.019 & 17370 \\
0.097 & 0.027 & 0.016 & 3580 \\
0.200 & 0.012 & 0.007 & 1216 \\
0.200 & 0.012 & 0.007 & 1205 \\
\hline
\end{tabular}

Table 2. Optimized combinations of $|\mathbf{G}|, \delta$ and $\Delta$ from the Rician CRLB objective function.

function based on the Gaussian CRLB and Table 2 shows the optimized scheme for the Rician CRLB.

The Gaussian CRLB objective function tends to favour higher levels of diffusion weighting that results in measurements with very low signal to noise. The Rician version penalizes high diffusion weighting more. The difference is marked in this example where $R$ is close to the limit of the measurable range (see later sections). At higher $R$, optimized schemes become similar for the two noise models. Simulations (not shown) demonstrate clear superiority of acquisition schemes optimized using the Rician CRLB and we shall not consider the Gaussian CRLB further.

\subsection{Choice of $M$}

This section compares different combinations of $N$ and $M$. We optimize the acquisition separately for each $R \in$ $\{1,2,5,10,20\} \mu \mathrm{m}$ with each $M \in\{2,3,4,5,6,8,10,12\}$ and $N=120 / M$. We then compute the value of the objective function, $F$ in (8), for each $\mathbf{n} \in S_{500}$. Figure 2 plots the mean of those 500 values of $F$ for each combination of $R$ and $M$ at each $G_{\max }$. The CRLB is lowest for $R=5 \mu \mathrm{m}$, but comparable for 10 and $20 \mu \mathrm{m}$ and, for $G_{\max }=0.2 \mathrm{~T} \mathrm{~m}^{-1}, 2 \mu \mathrm{m}$. The objective function is much higher (off the graph scale) for $R=1 \mu \mathrm{m}$, suggesting that lower $R$ is harder to estimate. Values of $M \in\{3,4,5,6\}$ give the lowest $F$ and $M=4$ is best most often. We use $N=30, M=4$ for all the remaining experiments.

\subsection{Axon radius estimation}

Next we use simulation experiments to check the accuracy with which we can recover known parameter settings using the optimized acquisition schemes. In all the simulation experiments, we set all the model parameters to the values listed in section 3.2.2 and synthesize data from the model using the acquisition scheme we are testing. We
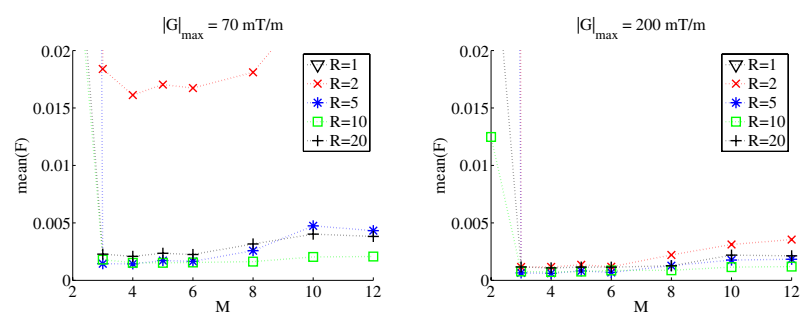

Figure 2. Plots of the mean $F$ over 500 orientations against $M$ at various $R$ for $G_{\max }$ of 0.07 (left) and $0.2 \mathrm{~T} \mathrm{~m}^{-1}$ (right).

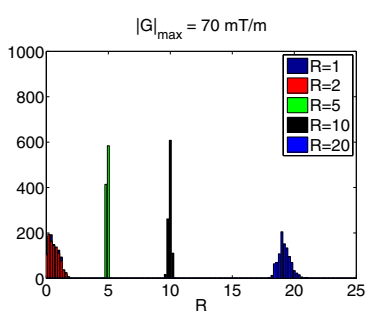

(a)

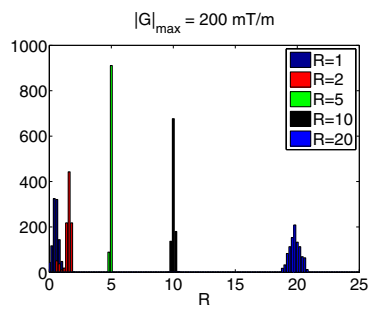

(b)
Figure 3. Histograms of samples drawn from posterior distributions on $R$. Experiments with each setting of $R$ use schemes optimized for that specific $R$.

add synthetic Rician noise with $\sigma_{0}=0.02$, to give an unweighted SNR of 50 if TE $=0.1 \mathrm{~s}$, which is achievable with sufficiently large voxel size. We use $\mathrm{T}_{2}=0.07 \mathrm{~s}$ to estimate a corrected SNR using the TE required for each specific acquisition scheme.

We use a simple Markov Chain Monte Carlo (MCMC) procedure to examine the posterior distribution of the model parameters given the data. We use broad uniform priors for all the scalar model parameters. The prior on $\mathbf{n}$ is uniform on the sphere. The prior on the noise-level parameter $\sigma$ is a broad uninformative Gamma distribution. Proposal distributions are zero-mean Gaussians with standard deviations chosen manually to give suitable acceptance rates. Samples of the model parameters come from a Metropolis-Hastings sampler; samples of $\sigma$ from Gibbs sampling. We set the initial parameters to the known correct values to speed up convergence. We use a burn-in period of 10,000 iterations, which ensures convergence, and a sampling interval of 1000, which gives approximately independent samples. We gather 100 samples in each experiment.

Figure 3 plots histograms of samples of the posterior distributions on $R$ for each of the various true $R$ at each $G_{\max }$. For each histogram, we run ten separate MCMC experiments each with a different setting of $\mathbf{n}$ and independent noise, so the total number of samples is 1000. Each experiment uses the acquisition scheme optimized for that specific value of $R$, which should give the best possible chance of recovering $R$ precisely and accurately. 


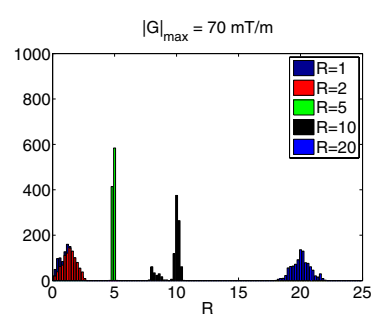

(a)

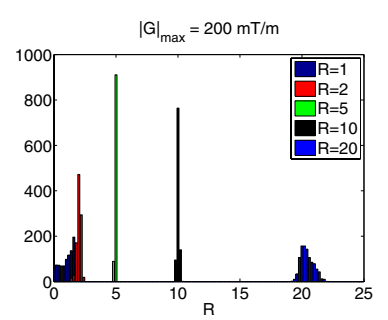

(b)
Figure 4. As figure 3, but experiments schemes optimized only for $R=5 \mu \mathrm{m}$.

The axon radius is easiest to estimate when it is in the range $[5,10] \mu \mathrm{m}$. Variance of the posterior increases as $R$ increases or decreases from this range. For $G_{\max }=$ $0.07 \mathrm{~T} \mathrm{~m}^{-1}, R=1 \mu \mathrm{m}$ and $R=2 \mu \mathrm{m}$ are identifiable as $R \in[0,2] \mu \mathrm{m}$, but are indistinguishable from each other and smaller radii. However, at $G_{\max }=0.2 \mathrm{~T} \mathrm{~m}^{-1}$, the posterior distributions when $R=1,2 \mu \mathrm{m}$ separate and are centred on the correct values. The results in figure 3 are an upper bound on what is achievable, since they come from the acquisition optimized individually for each $R$. For comparison, figure 4 shows similar results using the same acquisition sequence (that optimized for $R=5 \mu \mathrm{m}$ ) throughout the experiments with each $G_{\max }$. Histograms are slightly broader, but the trends remain similar. Further improvements should come from optimizing $F$ averaged over the range of $R$.

\section{Discussion}

In summary, we have discussed models that relate the diffusion MRI signal directly to parameters of the tissue microstructure that are potentially useful biomarkers. We have constructed a simple geometric model of white matter that relates parameters such as the axon radius and density to the diffusion MRI signal. We have developed a novel method based on the CRLB to optimize the experiment design for fitting the model parameters in vivo where the axon orientation is unknown. The experiment design technique does not depend on the specific model and will work with any model that relates the diffusion MRI signal to microstructural parameters of interest. In particular, it could also provide optimal acquisition schemes for DT-MRI, which are not yet agreed. Experiments here test the feasibility of measuring axon radii in vivo using the optimized acquisition schemes. Results suggest that, on a modern human MRI scanner, we can distinguish bundles of axons with radii of 5,10 and $20 \mu \mathrm{m}$ from each other and from smaller radii, but axons with radii of $2 \mu \mathrm{m}$ or less are indistinguishable from each other. This suggests that fitting models of axon radius distributions, like that of [4] is feasible in vivo using a suitable acquisition scheme. As we increase the available gradient strength, smaller radii become distinguishable. At
$G_{\max }=0.2 \mathrm{~T} \mathrm{~m}^{-1}$, we can distinguish radii of 1 or $2 \mu \mathrm{m}$ from each other and from smaller radii, which covers the useful range for brain tissue.

Here, we focus only on one parameter of the model, which is the axon radius. The other model parameters are also potentially interesting biomarkers. In particular, the intra-cellular volume fraction, $f$, provides a measure of axon density. Although not shown here, the precisions with which we can estimate the other model parameters follow similar trends to $R$. Future work will evaluate the dependence of results on the other model parameters.

Further work is required for practical application of the work. The model we test here is very simple and may need to incorporate other effects, such as distributions of radii, permeability and compartmental diffusivity and $\mathrm{T}_{2}$, to provide realistic and useful biomarkers. However, the structure of brain tissue is so complex that any biomarker estimated in this way will always be an approximation. Even this simple model may provide crude in vivo measurements that correlate with cell dimensions closely enough to be useful. Current work focuses on implementing the optimized acquisition schemes for testing on human and animal models. Further work will also improve the optimization for experiment design, for example by averaging $F$ over appropriate prior distributions on the parameters, and further optimization of the MCMC fitting procedure is certainly possible. We also intend to investigate alternative pulse sequences for making diffusion MRI measurements, which may prove advantageous over PGSE.

\section{References}

[1] D. C. Alexander. An introduction to computational diffusion MRI: the diffusion tensor and beyond. In J. Weichert and H. Hagen, editors, Visualization and Image Processing of Tensor Fields. Springer, 2006. 2

[2] J. Andersson. Maximum likelihood estimation of diffusion parameters with a Rician noise model. In Proc. ISMRM, page 1881, Berlin, 2007. ISMRM. 5

[3] Y. Assaf and P. J. Basser. Composite hindered and restricted model of diffusion (CHARMED) MR imaging of the human brain. NeuroImage, 30:1100-1111, 2005. 3

[4] Y. Assaf, R. Z. Freidlin, and P. J. Basser. The measurement of the axon diameter distribution in white matter using diffusion MR methods. In Proc. ISMRM, page 842, Miami, 2005. ISMRM. 1, 3, 4, 7

[5] L. Avram, Y. Assaf, and Y. Cohen. The effect of rotational angle and experimental parameters on the diffraction pattern and micro-structural information obtained from $q$-space diffusion NMR: implication for diffusion in white matter fibers. Journal of Magnetic Resonance, 169:30-38, 2004. 3

[6] B. Balinov, B. Jonsson, P. Linse, and O. Soderman. The NMR self-diffusion method applied to restricted diffusion. simulation of echo attenuation from molecules in spheres and between planes. Journal of Magnetic Resonance. Series A., 104:17-25, 1993. 2 
[7] P. J. Basser, J. Matiello, and D. Le Bihan. MR diffusion tensor spectroscopy and imaging. Biophysical Journal, 66:259267, 1994. 1, 2

[8] T. E. J. Behrens and Johansen-Berg, H., et al. Non-invasive mapping of connections between human thalamus and cortex using diffusion imaging. Nature Neuroscience, 7:750-757, 2003. 1

[9] S. L. Bengtsson, Z. Nagy, S. Skare, L. Forsman, H. Forssberg, and F. Ullen. Extensive piano practicing has regionally specific effects on white matter development. Nature Neuroscience, 8:1148-1150, 2005. 1

[10] P. T. Callaghan. Principles of Magnetic Resonance Microscopy. Oxford, UK: Oxford Science Publications, 1991. 2

[11] P. T. Callaghan. Pulsed-gradient spin-echo NMR for planar, cylindrical and spherical pores under conditions of wall relaxation. Journal of Magnetic Resonance, 113:53-59, 1995. 2

[12] P. T. Callaghan, C. D. Eccles, and Y. Xia. NMR microscopy of dynamic displacements: $k$-space and $q$-space imaging. Journal of Phys. E. Sci. Instrum., 21:820, 1988. 1, 3

[13] R. M. Henkelman. Measurement of signal intensities in the presence of noise in MR images. Medical Physics, 12:232233, 1985. 5

[14] K. M. Jansons and D. C. Alexander. Persistent angular structure: new insights from diffusion MRI data. Inverse Problems, 19:1031-1046, 2003. 4

[15] D. K. Jones, M. Catani, C. Pierpaoli, S. J. Reeves, S. S. Shergill, and M. O'Sullivan. Age effects on diffusion tensor magnetic resonance imaging tractography measures of frontal cortex connections in schizophrenia. Human Brain Mapping, 27:230-238, 2005. 1

[16] D. K. Jones, M. A. Horsfield, and A. Simmons. Optimal strategies for measuring diffusion in anisotropic systems by magnetic resonance imaging. Magnetic Resonance in Medicine, 42:515-525, 1999. 4

[17] L. L. Latour, K. Svoboda, P. P. Mitra, and C. H. Sotak. Timedependent diffusion of water in a biological model system. Proc. Nat. Acad. Sci., 91:1229-1233, 1994. 3

[18] B. W. Lindgren. Statistical Theory. Chapman and Hall, New York, 1993. 4

[19] R. C. McKinstry and Mathur, A. et al. Radial organization of developing preterm human cerebral cortex revealed by noninvasive water diffusion anisotropy MRI. Cerebral Cortex, 12:1237-1243, 2002. 1

[20] P. P. Mitra and B. I. Halperin. Effects of finite gradientpulse widths in pulsed-field-gradient diffusion measurements. Journal of Magnetic Resonance, 113:94-101, 1995. 2

[21] J. Murday and R. Cotts. Self-diffusion coefficient of liquid lithium. Journal of Chemical Physics, 48:4938-4945, 1968. 2,3

[22] C. H. Neuman. Spin echo of spins diffusing in a bounded medium. Journal of Chemical Physics, 60:4508-4511, 1974. 2

[23] J. M. Newton, N. S. Ward, G. J. M. Parker, R. Deichmann, D. C. Alexander, K. J. Friston, and R. S. J. Frackowiak. Noninvasive mapping of corticofugal fibres from multiple motor areas - relevance to stroke recovery. Brain, 127:1844-1858, 2006. 1

[24] C. Nicholson. Diffusion and related transport mechanisms in brain tissue. Reports on progress in physics, 64:815-884, 2001. 2, 4

[25] G. J. M. Parker and Luzzi, S. et al. Non-invasive structural mapping of two auditory-language pathways in the human brain. NeuroImage, 24:656-666, 2005. 1

[26] A. Pfefferbaum, E. V. Sullivan, M. Hedehus, K. O. Lim, E. Adalsteinsson, and M. Moseley. Age-related decline in brain white matter anisotropy measured with spatially corrected echo-planar diffusion tensor imaging. Magnetic Resonance in Medicine, 44:259-268, 2000. 1

[27] H. W. R. Powell and Parker, G. J. M. et al. MR-tractography predicts visual field defects following temporal lobe resection. Neurology, 64:596-599, 2005. 1

[28] P. N. Sen and P. J. Basser. A model for diffusion in white matter. Biophysical Journal, 89:2927-2938, 2005. 3

[29] O. Soderman and B. Jonsson. Restricted diffusion in cylindrical geometry. Journal of Magnetic Resonance A, 117:9497, 1995. 2

[30] C. H. Sotak. The role of diffusion tensor imaging in the evaluation of ischemic brain injury - a review. NMR in Biomedicine, 15:561-569, 2002. 1

[31] G. J. Stanisz, A. Szafer, G. A. Wright, and M. Henkelman. An analytical model of restricted diffusion in bovine optic nerve. Magnetic Resonance in Medicine, 37:103-111, 1997. $1,2,3,4$

[32] A. Szafer, J. Zhong, and J. C. Gore. Theoretical model for water diffusion in tissues. Magnetic Resonance in Medicine, 33:697-712, 1995. 3

[33] T. Toosy and Werring, D. J. et al. Diffusion tensor imaging detects corticospinal tract involvement at multiple levels in amyotrophic lateral sclerosis. J. Neurol. Neurosurg. Psychiatry, 74:1250-1257, 2003. 1

[34] D. S. Tuch, D. H. Salat, J. J. Wisco, A. K. Zaleta, N. D. Hevelone, and H. D. Rosas. Choice reaction time performance correlates with diffusion anisotropy in white matter pathways supporting visuospatial attention. Proc. Nat. Acad. Sci., 102:12212-12217, 2005. 1

[35] P. Van Gelderen, D. DesPres, P. C. M. van Zijl, and C. T. W. Moonen. Evaluation of restricted diffusion in cylinders. phosphocreatine in rabbit leg muscle. Journal of Magnetic Resonance Series B, 103:255-260, 1994. 2, 3

[36] Various authors. Special issue on neurological and psychiatric disease. Neuron, 52(1), 2006. 1

[37] J.-C. Weng, J.-H. Chen, L.-W. Kuo, V. J. Wedeen, and W.Y. Tseng. Maturation-dependent microstructure length scale in corpus callosum of fixed rat brain by magnetic resonance diffusion-diffraction. Magnetic Resonance Imaging, 25:7886, 2007. 1,3

[38] I. Zelinka. SOMA - self organizing migrating algorithm. In B. B. V. and O. G., editors, New Optimization Techniques in Engineering. Springer, 2004. 5 